\title{
Correction: Emeakaroha, A. et al. Challenges in Improving Energy Efficiency in a University Campus through the Application of Persuasive Technology and Smart Sensors. Challenges 2012, 3, 290-318
}

\author{
Anthony Emeakaroha*, Chee Siang Ang and Yong Yan \\ School of Engineering and Digital Arts, University of Kent, Canterbury CT2 7NT, UK; \\ E-Mails: C.S.Ang@Kent.ac.uk (C.S.A.); y.yan@Kent.ac.uk (Y.Y.) \\ * Author to whom correspondence should be addressed; E-Mail: ae226@Kent.ac.uk; \\ Tel.: +44-122-782-3458; Fax: +44-122-745-6084.
}

Received: 20 January 2013 / Accepted: 30 January 2013 / Published: 5 February 2013

It has come to our attention that our paper "Challenges in Improving Energy Efficiency in a University Campus through the Application of Persuasive Technology and Smart Sensors" [1] contains some minor errors. Based on that we have done some minor corrections as stated below.

The affiliation information has been changed to: School of Engineering and Digital Arts, University of Kent, Canterbury CT2 7NT, UK.

In the abstract, we changed the word "grave" to "serious". We also removed the following sentences from the abstract: "However, the situation is different", "with the complexity in behaviour of our target groups", "the students who are living in the halls of residence", "there are economical and environmental aspects to be addressed in these issues, in the campus halls of residence".

In Section 3, the proposed system architecture description, we would like to remove the following sentence: "Due to the Hawthorns effects, the other previous interventions suffer from the lack of maintaining a constant and sustainable behaviour change in students. Based on these findings".

In Section 3.3 the following sentences were removed: "This is the reason why we proposed the combination of real-time energy feedback and appointed energy delegate so as to induce behaviour change on the students living in the halls of residences", "This is also where the effectiveness of appointing an energy delegate is lacking. Providing energy feedback is a critical foundation for any attempt to reduce energy consumption, and the feedback itself will likely curb energy usage to some extent. However, Sarah Darby points out that while feedback is critical for energy conservation behaviour, feedback alone is not always enough [14]. Other factors that lead to higher rates of energy 
conservation include contact with an advisor when needed, training, awareness and social infrastructure also plays a vital role".

In Section 5, the phrase "Initial Result" was replaced by "Initial Results of the Survey Study".

There are no changes for the rest of our paper. We apologize for the inconvenience this might have caused.

\section{Reference}

1. Emeakaroha, A.; Ang, C.S.; Yan, Y. Challenges in improving energy efficiency in a university campus through the application of persuasive technology and smart sensors. Challenges 2012, 3, 290-318.

(C) 2013 by the authors; licensee MDPI, Basel, Switzerland. This article is an open access article distributed under the terms and conditions of the Creative Commons Attribution license (http://creativecommons.org/licenses/by/3.0/). 